\title{
Human sputum interleukin-6 by exposure to PM10 among bus drivers in Klang Valley.
}

\begin{abstract}
Epidemiologic evidences suggest a positive association between elevated levels of Particulate Matter (PM) pollution and respiratory illness including acute and chronic lung diseases and respiratory symptoms. This study aimed to investigate the association between particulate matter 10 (PM10) and induction of inflammatory mediators; Interleukin-6 (IL-6). A total of 56 bus drivers as exposed group and 56 administrative staff as comparative group in Klang Valley were involved in this study. Exposure of PM10 was measured using Dust Trak Aerosol Monitor while ELISA technique was used for the analysis of IL-6. Questionnaire adapted from American Thoracic Society (ATS) was used to collect information on respondent's socio-economic status, working history, and respiratory symptoms. The results of socioeconomic status were showed that there were no significant differences found between the groups. The exposure level of PM10 were significantly higher among the bus drivers $(t=7.57, p<0.001)$ than to comparative group which the PM10 was almost 4 times higher in bus drivers. Similarly, the concentrations of IL-6 were also found significantly higher among bus drivers with $(\mathrm{z}=-2.28, \mathrm{p}<0.05)$. A positive correlation was observed between the PM10 and IL-6 among the exposed and the comparative group of the present study. Moreover, prevalence of respiratory symptoms among the bus drivers was significantly higher compared the administrative staff. In conclusion, the bus drivers have higher level of IL-6 and at higher risk for respiratory symptoms compared to comparative group due to the exposure to PM10.
\end{abstract}

Keyword: Bus driver; IL-6; Pm10; Respiratory symptoms. 